\title{
¿CÓMO DESCANSA EL PACIENTE ONCOHEMATOLÓGICO EN EL HOSPITAL?
}

\author{
HOW DOES THE ONCOHAEMATOLOGICAL PATIENT REST IN THE HOSPITAL?!
}

*Penas Casals, E., *Ruiz Navas, S., **Tormo Gasa, S.

*Enfermera de la Unidad de Hematología. * * Enfermera de la Unidad de On cología. Hospital Universitario Arnau de Vilanova. Lleida.

Palabras clave: Patrón reposo/ sueño; enfermo oncohematológico; factores perturbadores del sueño. Keywords: Rest/Sleep Model, Oncohematologic Patient, and Sleep Disturbing Factors.

\begin{abstract}
RESUMEN
El descanso nocturno es imprescindible para el bienestar físico y psíquico de toda persona y una inadecuada cobertura de esta necesidad influye negativamente en el paciente y altera su calidad de vida. El insomnio definido como "una deficiencia persistente en la cantidad del sueño que afecta a la normal actividad y bienestar del individuo" es una queja frecuente entre los pacientes hospitalizados; motivo que justifica la necesidad de una atención especial a este problema.
\end{abstract}

Nos planteamos como objetivos identificar qué factores afectan en el descanso nocturno y saber si el personal de enfermería conoce estos factores.

Se trata de un estudio descriptivo transversal realizado en las unidades de hematología y oncología durante el mes de marzo de 2008. Para recoger los datos elaboramos una encuesta para los pacientes y otra para el personal de enfermería.

El $91 \%$ de la muestra respondió que se despertaba durante la noche en el hospital y el $40 \%$ lo hace más de tres veces. Los factores que más alteran el descanso del paciente son los factores psicológicos (preocupación por la enfermedad, por la familia y el aburrimiento durante la hospitalización).

Como conclusiones destacamos que tanto enfermería como pacientes coinciden en que no se duerme en el hospital. El personal de enfermería demostró tener una buena percepción de las causas principales de insomnio de los pacientes. Ambos han coincidido en cómo los factores psicológicos influyen en el patrón de reposo/sueño.

Por ello sería importante que el personal de enfermería dedicara especial atención al manejo de los factores psicológicos a través de distintas técnicas. 


\section{ABSTRACT}

Resting is essential for physical and mental well being of everyone and inadequate coverage of this requirement negatively affects the patient and disturbs life quality. Insomnia, defined as "a persistent weakness in the quantity of sleep that affects the normal activity and well being of the individual," is a common complaint among hospitalized patients; a reason that justifies the need of special attention to this problem.

Our objectives were to identify factors that affect rest, and whether the nursing staff is aware of these factors.

This is a descriptive cross-sectional study conducted in the hematology and oncology units during the month of March 2008. To collect the data we created a survey for patients and for nurses.

A percentage of $91 \%$ of the sample responded that they woke up during the night in the hospital and $40 \%$ do so more than three times. The factors that affect the rest of the patient are the psychological factors (concern about the disease, the family, and boredom during hospitalization).

To conclude it is emphasized that both patients and nurses agree that a high percentage do not sleep in the hospital. The nursing staff showed a good perception of the major causes of insomnia in patients. Both have agreed on the extent to which psychological factors influence the pattern of rest/sleep.

Therefore it is important that the nursing staff devote special attention to the management of psychological factors through different techniques.

\section{INTRODUCCIÓN}

Durante todo el día el cuerpo consume gran cantidad de energía y el ser humano cuenta con varios medios para reparar este desgaste. Uno de estos medios es el descanso y el sueño. De hecho el descanso nocturno es imprescindible para el bienestar físico y psíquico de toda persona y una inadecuada cobertura de esta necesidad influye negativamente en las actividades de la vida diaria del paciente y altera de forma importante su calidad de vida.

El insomnio, definido como "una deficiencia persistente en la cantidad del sueño que afecta a la normal actividad y bienestar del individuo", es una queja frecuente entre los pacientes hospitalizados.

Aunque el insomnio no es considerado un problema prioritario sobretodo en oncología, tenemos que tener en cuenta que lo padecen un $50 \%$ de los enfermos con cáncer avanzado.

En el III Simposio Internacional de Cáncer y Calidad de Vida que se celebró en Sitges en 2007 se señaló que las causas que pueden ocasionar insomnio asociado al cáncer son múltiples y que abarcan desde los efectos psicológicos del diagnóstico, el mal control de los síntomas, una inadecuada higiene del sueño y la asociación de ansiedad y depresión hasta los efectos secundarios del tratamiento.

Sin embargo, un aspecto llamativo relacionado con este problema es la dificultad de los pacientes en la comunicación de sus deficiencias de sueño por considerarlo secundario en el contexto de su enfermedad.

Está demostrado que la deprivación del sueño puede producir alteraciones al hablar, irritabilidad, desorientación, cambios de humor, falta de colaboración.. El reposo por otra parte implica una liberación de las tensiones emocionales del SNC y una mejoría de las condiciones psíquicas del individuo. 
Por todo esto el mantenimiento de un sueño normal es valorado como uno de los elementos principales para determinar la calidad de vida y su efecto positivo en la capacidad de recuperación justifica la necesidad de una atención especial a este problema.

\section{1. - OBJETIVOS}

Los objetivos del estudio son:

$>$ Identificar qué factores afectan en el descanso nocturno en los pacientes oncohematológicos hospitalizados

$>$ Conocer que factores, según enfermería, perturban el sueño de los pacientes oncohematológicos

> Comparar si enfermería conoce los factores que alteran el descanso-sueño del paciente.

$>$ Establecer estrategias de actuación con el fin de mejorar la calidad del sueño del paciente.

\section{2. - MATERIAL Y MÉTODOS}

Es un estudio descriptivo transversal realizado en el Hospital Universitario Arnau de Vilanova de Lleida, hospital de referencia de segundo nivel de la región sanitaria de Lleida .El trabajo se ha realizado en las unidades de hematología y oncología durante el mes de marzo del 2008.

El servicio de hematología consta 26 camas y el de oncología de 14 camas.

Para recoger los datos elaboramos una encuesta para los pacientes (anexo !) y otra para el personal de enfermería (anexo !!), en la que se recogían datos personales y datos sobre los hábitos de sueño; en otro apartado se recogían los distintos factores de riesgo que pueden alterar el sueño.

Los factores de riesgo de la alteración del descanso y sueño se redactaron según los diagnósticos de enfermería de la NANDA, agrupándose en varios ítems valorados cada uno del 0 al 10 para que se pudieran puntuar fácilmente en qué grado les afectaba.

Para analizar los datos agrupamos los valores de la siguiente forma: 0-1: Nada. el factor no les afecta en el sueño; 2-4: Poco; 5-7: Bastante; 8-10: Mucho.

La muestra está formada por todos los pacientes que ingresaron en las unidades de hematología y oncología durante el periodo de estudio, excepto aquellos pacientes con deterioro cognitivo, demencia y pacientes en situación agónica.

Se les proporcionó la encuesta a los 3 días de ingreso para que el estrés del ingreso no afectara en los resultados. Se les explicó que era anónima y voluntaria, cómo debían rellenar cada ítem así como donde debían depositarla una vez contestada.

Se les informó que una vez contestada debían depositarla en el buzón habilitado en el control de enfermería.

Paralelamente realizamos una sesión informativa al personal de enfermería donde explicábamos el proyecto del estudio. Aprovechamos la ocasión para repartir las encuestas 
a auxiliares y enfermeras para investigar si éstas/éstos conocen realmente los motivos de la alteración del sueño/descanso del paciente ingresado.

Para el tratamiento de los datos se ha utilizado el programa Microsoft Excel 2003, realizando una estadística descriptiva de los datos obtenidos.

\section{3.- RESULTADOS}

\section{A. 1.- DESCRIPCIÓN DE LA MUESTRA DE LOS PACIENTES}

Se recogieron 64 encuestas. El $58 \%$ eran pacientes de hematología y el $42 \%$ de oncología (tabla 1, gráfico 1).

\section{Tabla 1}

\begin{tabular}{|l|l|l|l|}
\hline Servicio & Hematold Oncologia & Total \\
\hline Servicio & 37 & 27 & 64 \\
\hline$\%$ Resultados & $58 \%$ & $42 \%$ & $100 \%$ \\
\hline
\end{tabular}

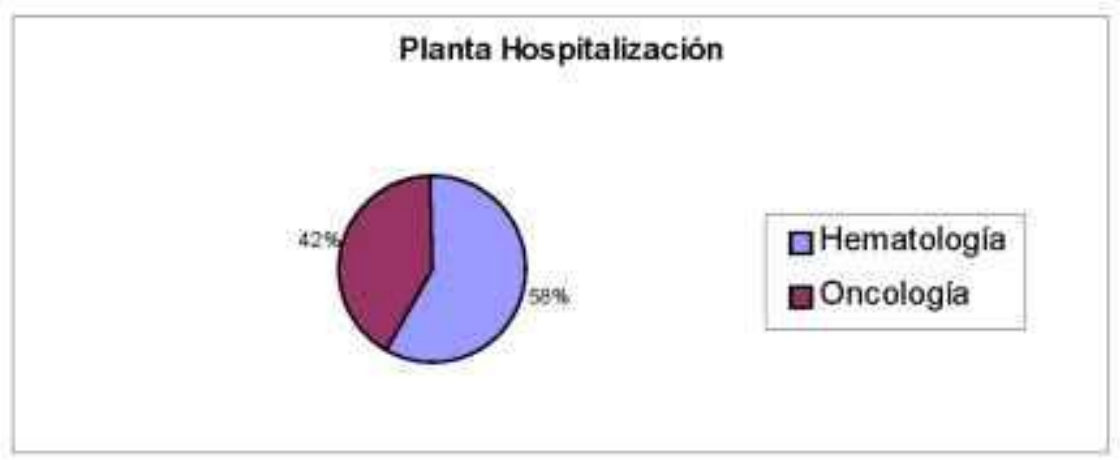

\section{Gráfico 1}

El $91 \%$ de la muestra respondió que se despertaba durante la noche (tabla 2, gráfico 2).

Tabla 2

\begin{tabular}{|l|l|l|l|}
\hline Despierta & SI & NO & Total \\
\hline Despierta noche & 58 & 6 & 64 \\
\hline$\%$ Resultados & $91 \%$ & $9 \%$ & $100 \%$ \\
\hline
\end{tabular}




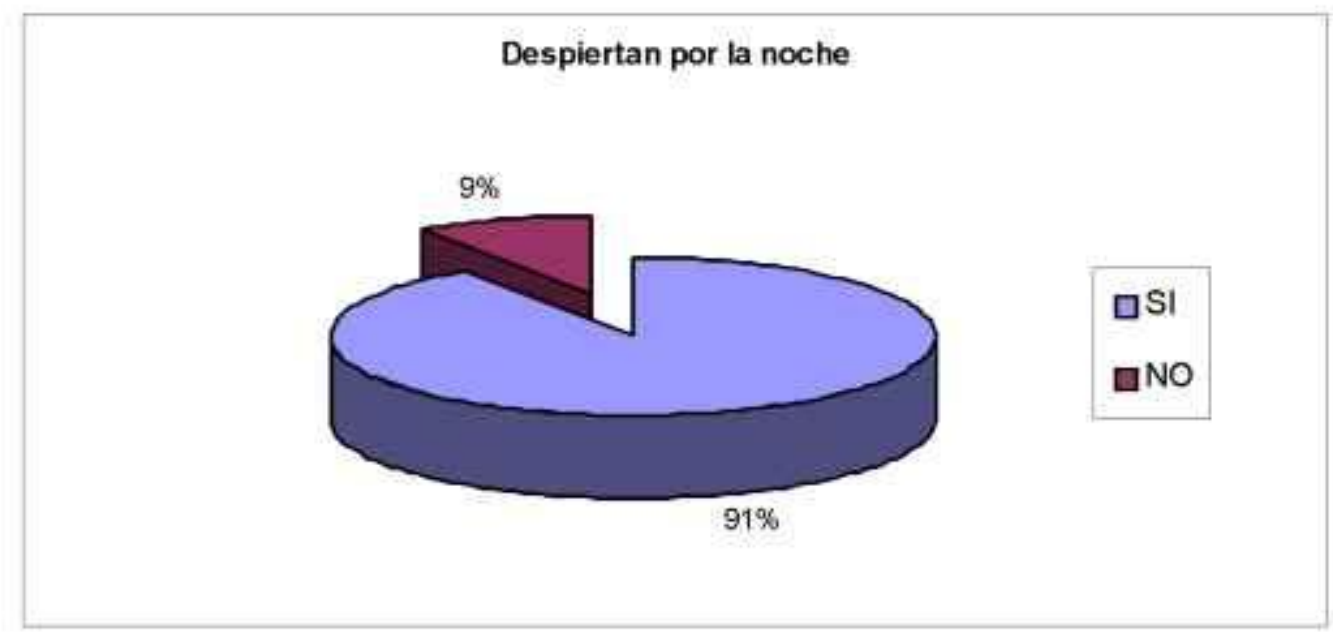

Gráfico 2

El 34\% dormía de 3-5 horas en el hospital; un $38 \%$ de 5 a 7 horas y un $28 \%$ más de 7 horas. En cambio en casa el 53,1\% duerme más de 7 horas. (tabla 3, gráfico 3 ).

Tabla 3

\begin{tabular}{|c|c|c|c|c|}
\hline Descanso & 3 -5Horas & $5-7$ Hore & $>7$ Hora & Total \\
\hline Casa & 5 & 25 & 34 & 64 \\
\hline$\%$ casa & $7,8 \%$ & $39,1 \%$ & $53,1 \%$ & $100,0 \%$ \\
\hline Hospital & 22 & 24 & 18 & 64 \\
\hline \%Hospital & $34 \%$ & $38 \%$ & $28 \%$ & $100 \%$ \\
\hline
\end{tabular}

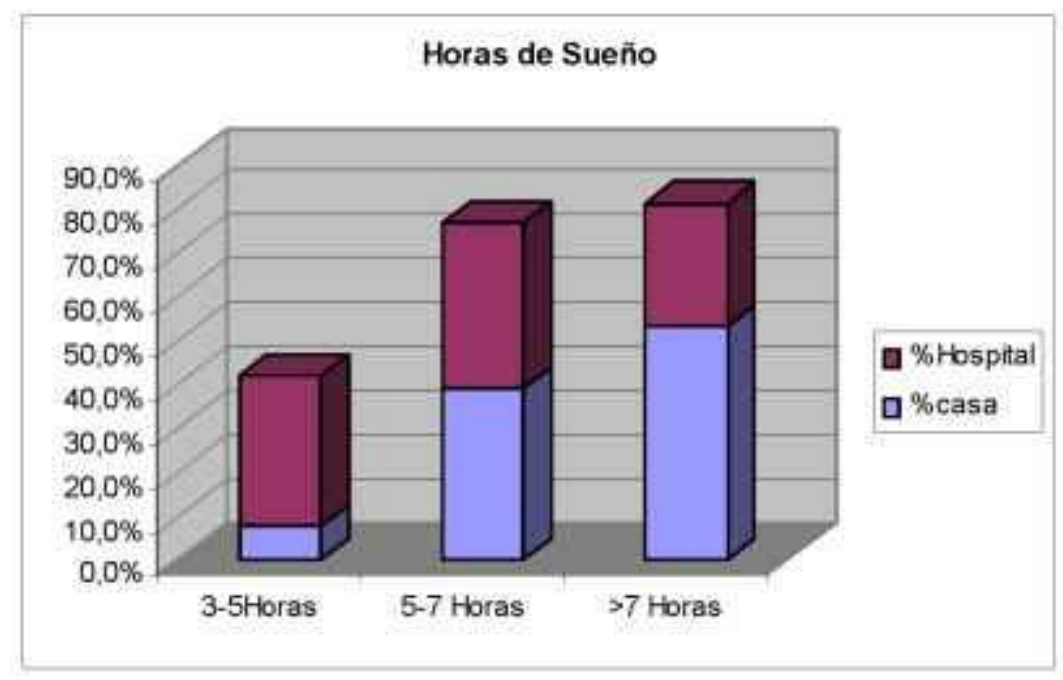

\section{Gráfico 3}

Respecto al número de veces que se despierta durante la noche, el $40 \%$ lo hace más de tres veces y el $26 \%$ lo hace de 2 a 3 veces. (tabla4, gráfico 4 ). 


\section{Tabla 4}

\begin{tabular}{|l|l|l|l|l|l|}
\hline $\mathbf{N}^{\circ}$ Veces & $0-1$ Veces & $1-2$ veces & $2-3$ veces & 3 Veces & Total \\
\hline Despierta noche 7 & 13 & 15 & 23 & 58 \\
\hline \%Resultados & $12 \%$ & $22 \%$ & $26 \%$ & $40 \%$ & $100 \%$ \\
\hline
\end{tabular}

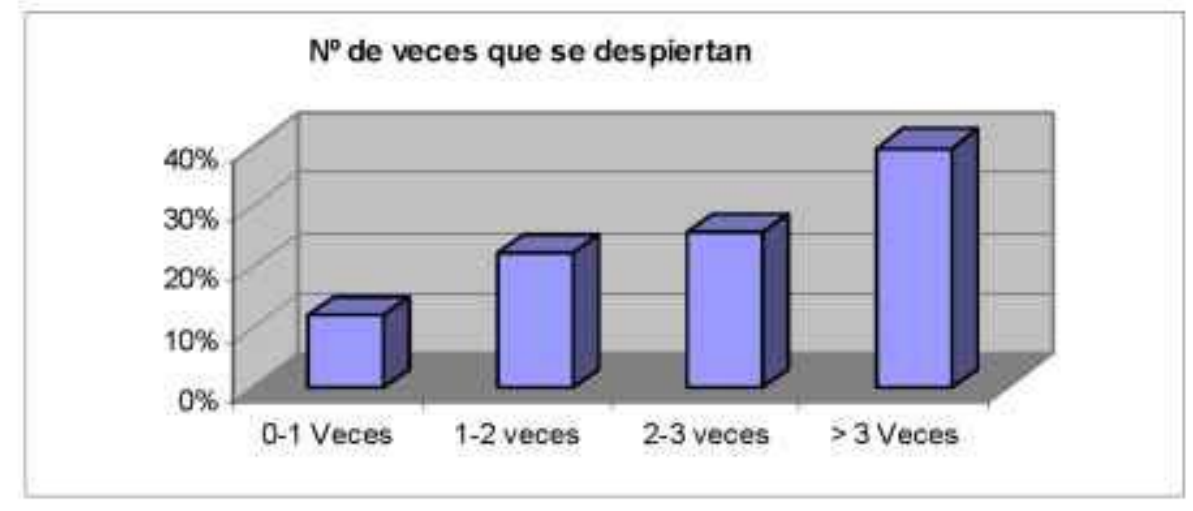

\section{Gráfico 4}

El $61 \%$ de la muestra dormía durante el día y un $39 \%$ de ésta lo hacia de una a dos horas al día (tabla 5 y 6 , gráfico 5 y 6 ).

\section{Tabla 5}

\begin{tabular}{|l|l|l|l|}
\hline Duerme de dia & SI & NO & Total \\
\hline Resultados & 39 & 25 & 64 \\
\hline \%Resultados & $61 \%$ & $39 \%$ & \\
\hline
\end{tabular}

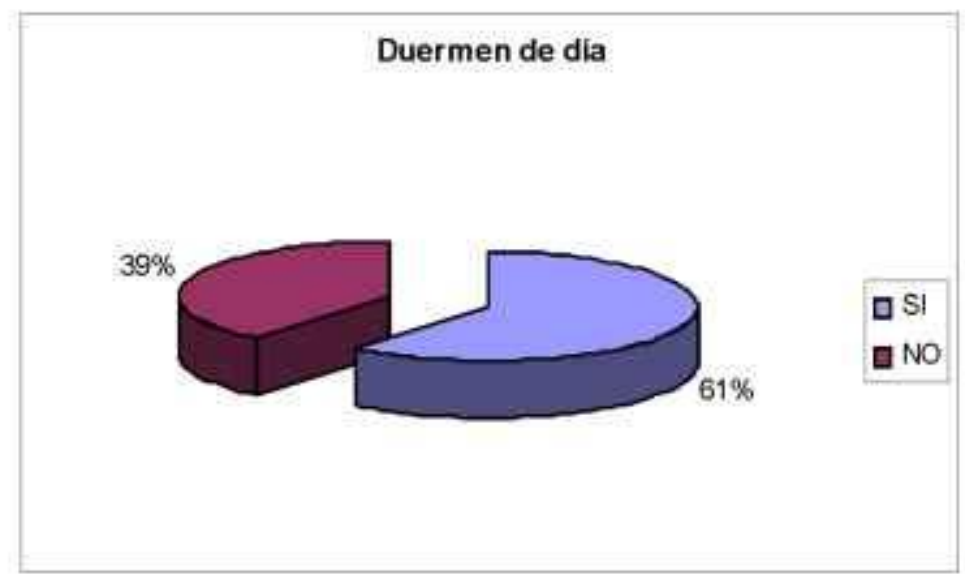

Gráfico 5 
Tabla 6

\begin{tabular}{|c|c|c|c|c|}
\hline Horas & 30 '- $\mathrm{h}$ & $1 \mathrm{~h}-2 \mathrm{~h}$ & $2 \mathrm{~h}-4 \mathrm{~h}$ & Total \\
\hline Duerme de di & 11 & 23 & 5 & 39 \\
\hline$\%$ Resultado & $28 \%$ & $59 \%$ & $13 \%$ & $100 \%$ \\
\hline
\end{tabular}

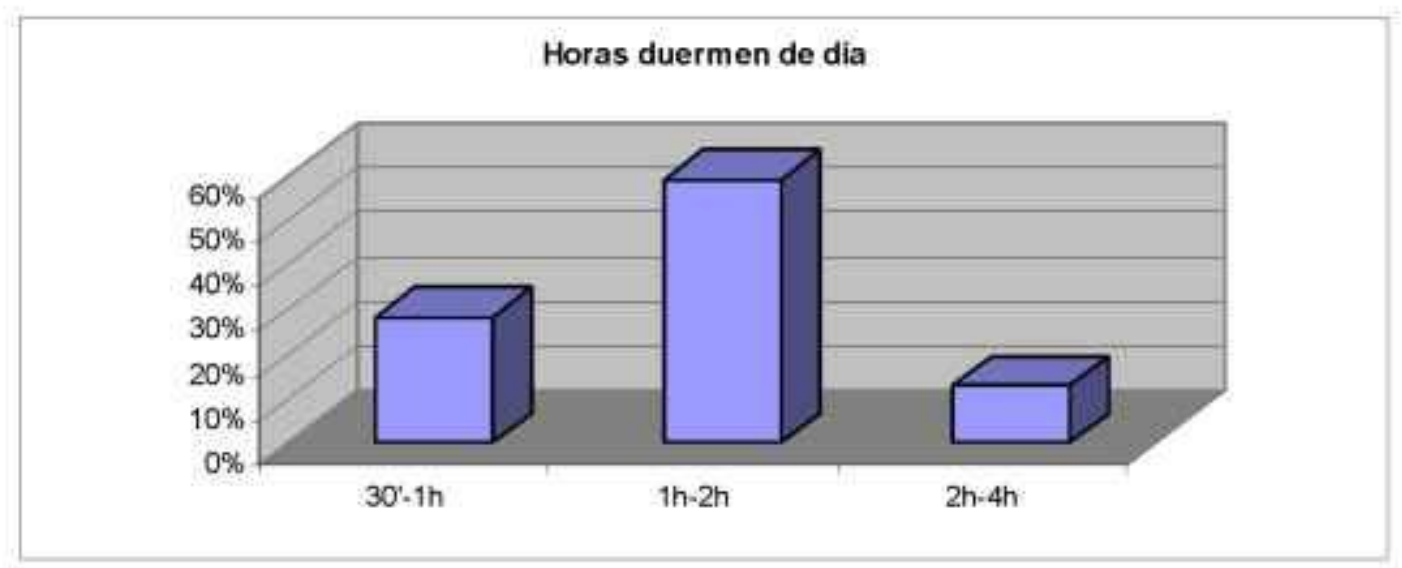

Gráfico 6

El $84 \%$ de los pacientes realizan actividades antes de ir a dormir; tienen el hábito de ver la TV un $63 \%$ y leen el $44 \%$. (tabla 7 y 8 , gráfico 7 y 8 ).

Tabla 7

\begin{tabular}{|l|l|l|l|}
\hline Actividades & SI & NO & Total \\
\hline Actividades & 54 & 10 & 64 \\
\hline$\%$ Resultados & $84 \%$ & $16 \%$ & $100 \%$ \\
\hline
\end{tabular}

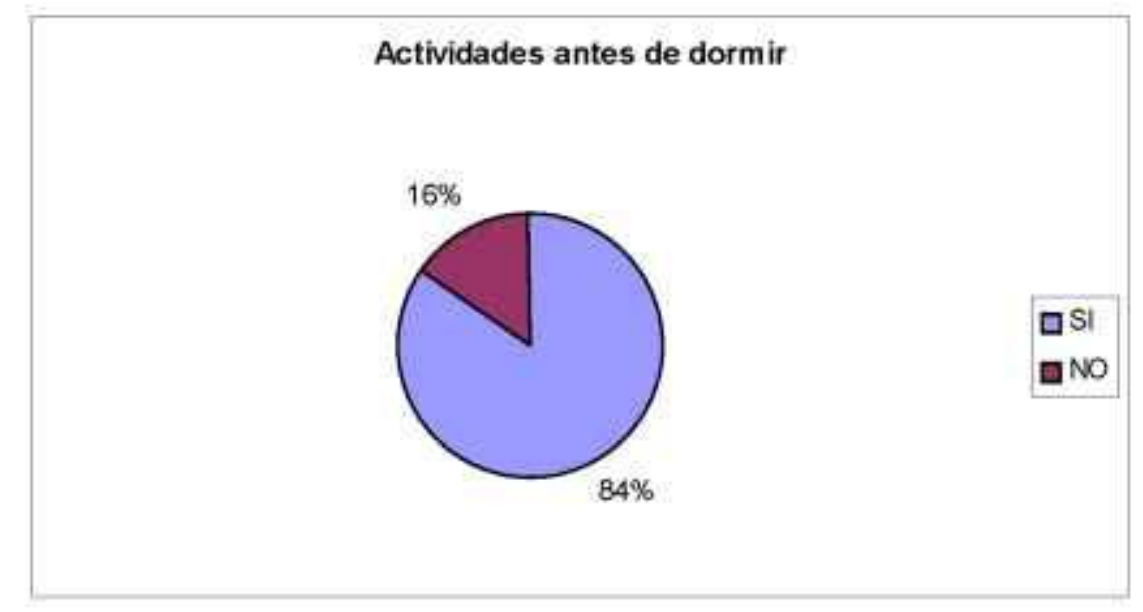

Gráfico 7 


\section{Tabla 8}

\begin{tabular}{|r|c|c|c|c|c|}
\hline Actividad & Rádio & TV & Leer & Ordenador & otras \\
\hline Actividades & 13 & 40 & 28 & 7 & 2 \\
\hline$\%$ Resultados & $20 \%$ & $63 \%$ & $44 \%$ & $11 \%$ & $3 \%$ \\
\hline
\end{tabular}

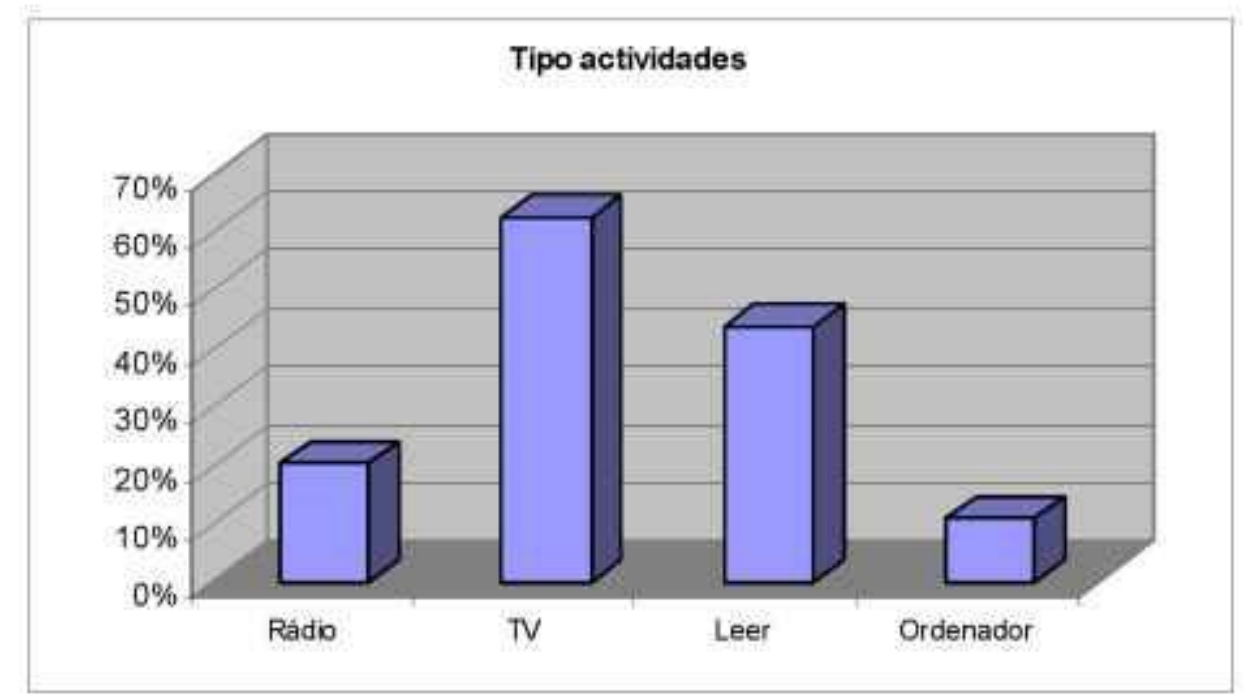

\section{Gráfico 8}

\section{B.1.- DESCRIPCIÓN DE LOS FACTORES QUE PERTURBAN EL SUEÑO. FACTORES AMBIENTALES}

Sobre la realización de los procedimientos de enfermería (toma de TA, administrar medicación, curas...) durante la noche, al $31 \%$ no les afectaba nada y el $36 \%$ respondió que les afectaba bastante en el sueño.

En relación al ruido, el $34 \%$ no les molesta nada y el $31 \%$ les molesta bastante.

La incomodidad de la cama, la iluminación de la habitación y la temperatura de la habitación no les afecta nada en un $38 \%, 45 \%$ y un $38 \%$ respectivamente.

El hecho de compartir habitación no les afecta nada en un $41 \%$, un $25 \%$ mucho y un $23 \%$ bastante (tabla 9, gráfico 9).

\section{$\underline{\text { Factores de riesgo }}$}

Tabla 9

\begin{tabular}{|c|c|c|c|c|c|c|}
\hline Ambientales & Procedimien & Ruido & Incomodid & Iluminacid & Comparti & Temperati \\
\hline Nada & $31 \%$ & $34 \%$ & $38 \%$ & $45 \%$ & $41 \%$ & $38 \%$ \\
\hline Poco & $22 \%$ & $23 \%$ & $22 \%$ & $30 \%$ & $11 \%$ & $19 \%$ \\
\hline Bastante & $36 \%$ & $31 \%$ & $25 \%$ & $17 \%$ & $23 \%$ & $19 \%$ \\
\hline Mucho & $14,1 \%$ & $11 \%$ & $16 \%$ & $8 \%$ & $25 \%$ & $14 \%$ \\
\hline
\end{tabular}




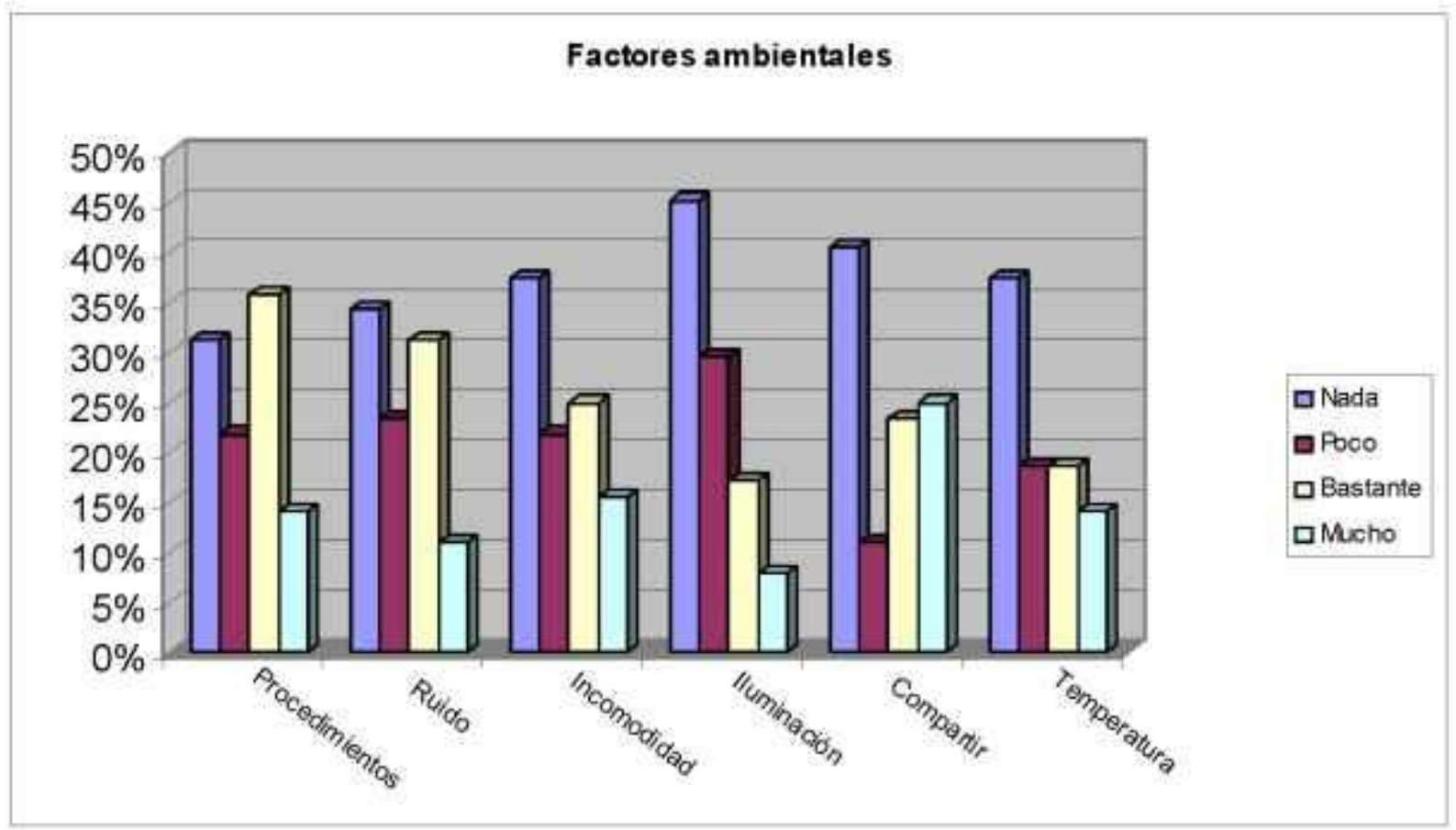

\section{Gráfico 9}

\section{FACTORES EXTERNOS}

El llevar sueros no perturba nada el descanso al $55 \%$ de los pacientes, el sondaje vesical tampoco al $83 \%$, la sonda nasogástrica no afecta nada al $84 \%$ y los drenajes tampoco al $80 \%$ de ellos.

Respecto al ser portador oxigenoterapia, ya sea en gafas nasales o mascarilla, no les afecta nada al $62 \%$.(tabla 10 y gráfico 10 ).

\section{Tabla 10}

\begin{tabular}{|l|l|l|l|l|l|}
\hline Factores Externo Suero & SV & SNG & Oxigeno & Drenajes \\
\hline Nada & $55 \%$ & $83 \%$ & $84 \%$ & $72 \%$ & $80 \%$ \\
\hline Poco & $17 \%$ & $8 \%$ & $6 \%$ & $13 \%$ & $8 \%$ \\
\hline Bastante & $17 \%$ & $6 \%$ & $6 \%$ & $5 \%$ & $9 \%$ \\
\hline Mucho & $9 \%$ & $3 \%$ & $3 \%$ & $0 \%$ & $3 \%$ \\
\hline
\end{tabular}




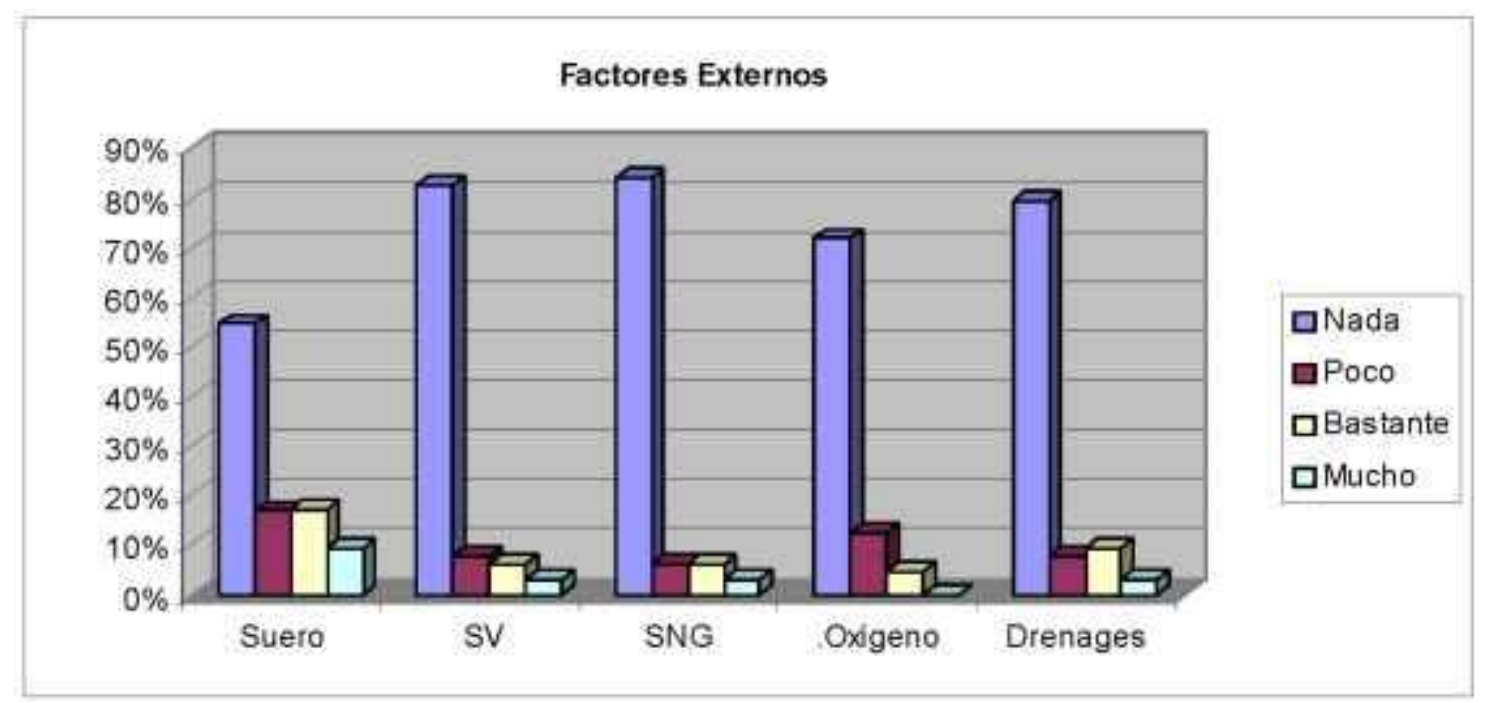

Gráfico 10

\section{FACTORES FISIOLÓGICOS}

El dolor afecta en nada a un $38 \%$, en poco a un $20 \%$ y mucho un $27 \%$; la micción nocturna no afecta nada al $55 \%$; la disnea no afecta nada a un $63 \%$ pero a un $21,9 \%$ les afecta bastante.

No les afecta en nada al descanso otros factores como las nauseas y vómitos (75\%), la fiebre $(64 \%)$, la tos $(56 \%)$, epigastrálgias $(59 \%)$, hambre/sed $(66 \%)$.

La movilidad en la cama no afecta nada al $45 \%$ y bastante al $25 \%$. (tabla 11 y gráfico 11 ).

\section{Tabla 11}

\begin{tabular}{|l|l|l|l|l|}
\hline Factores fisiológicos & Nada & Poco & Bastante & Mucho \\
\hline Dolor & $38 \%$ & $20 \%$ & $17,2 \%$ & $27 \%$ \\
\hline Micción & $55 \%$ & $14 \%$ & $18,8 \%$ & $11 \%$ \\
\hline Vómitos. & $75 \%$ & $6 \%$ & $6,3 \%$ & $13 \%$ \\
\hline Fiebre & $64 \%$ & $16 \%$ & $10,9 \%$ & $11 \%$ \\
\hline Tos & $56 \%$ & $16 \%$ & $12,5 \%$ & $16 \%$ \\
\hline Diseña & $63 \%$ & $86 \%$ & $21,9 \%$ & $6 \%$ \\
\hline Epigastralgias & $59 \%$ & $16 \%$ & $14,1 \%$ & $11 \%$ \\
\hline Movilizacion & $45 \%$ & $14 \%$ & $25,0 \%$ & $16 \%$ \\
\hline Hambre/ Sed & $66 \%$ & $19 \%$ & $10,9 \%$ & $5 \%$ \\
\hline
\end{tabular}




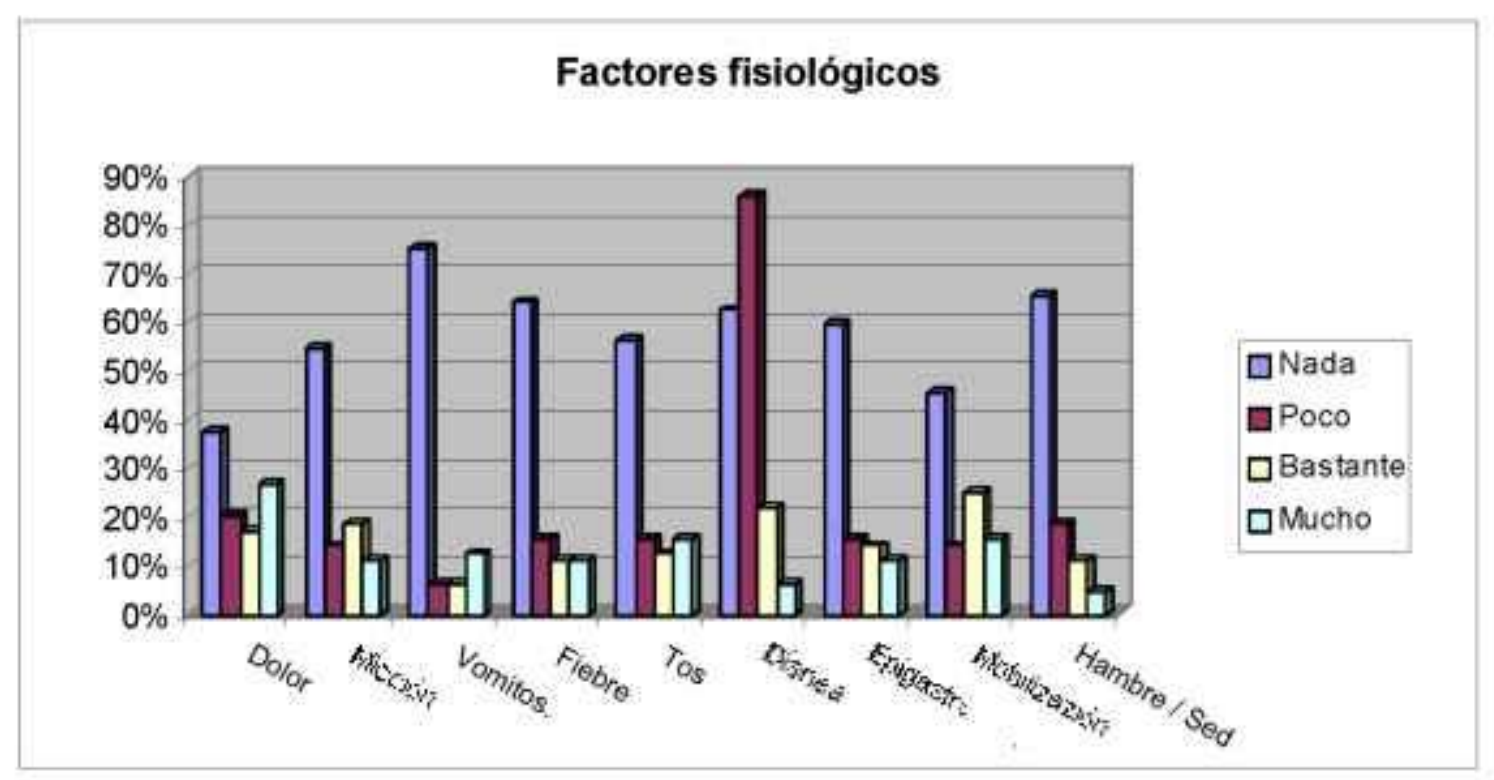

\section{Gráfico 11}

\section{FACTORES PSICOLÓGICOS}

La preocupación por la enfermedad y por la familia les afecta mucho al $44 \%$ y la preocupación por la enfermedad a un $34,6 \%$ les afecta bastante.

El trabajo no les afecta nada al $66 \%$.

La ansiedad/depresión afecta bastante al $36 \%$ y el $31 \%$ en nada.

El aburrimiento les perturba mucho el sueño al 33\% y bastante al $30 \%$ (tabla 12 y gráfico 12).

\section{Tabla 12}

\begin{tabular}{|l|l|l|l|l|}
\hline Factores Psicológicos & Nada & Poco & Bastante & Mucho \\
\hline Enfermedad & $9 \%$ & $12,5 \%$ & $34 \%$ & $43,8 \%$ \\
\hline Familia & $13 \%$ & $12,5 \%$ & $31 \%$ & $43,8 \%$ \\
\hline Trabajo & $66 \%$ & $6,3 \%$ & $14 \%$ & $14,1 \%$ \\
\hline Ansiedad Depresión & $31 \%$ & $21,9 \%$ & $36 \%$ & $10,9 \%$ \\
\hline Aburrimiento & $20 \%$ & $17,2 \%$ & $30 \%$ & $32,8 \%$ \\
\hline
\end{tabular}




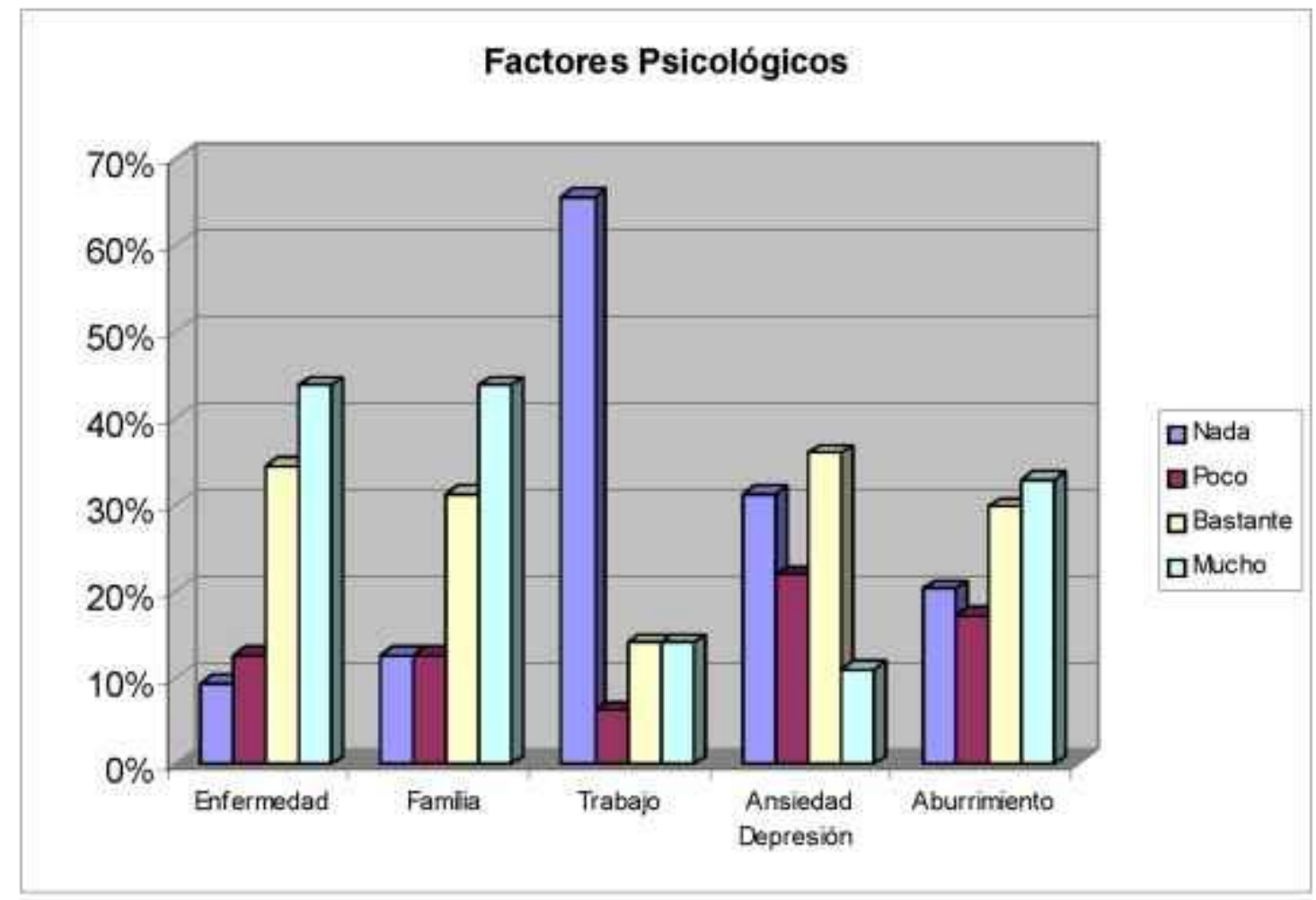

Gráfico 12

A.2.- DESCRIPCION DE LA MUESTRA DEL PERSONAL DE ENFERMERÍA

En relación a la muestra del personal de enfermería que respondió las encuestas, el $64,5 \%$ son enfermeras y el $41,9 \%$ auxiliares; de todas ellas, el $36 \%$ lo comprenden un grupo de edad de 20-30 años y un 24\% de 30-40 años.(tablas 13 y 14, gráfico 13 y 14 ).

\section{Muestra personal enfermería}

Tabla 13

\begin{tabular}{|l|l|l|l|}
\hline & DUI & Auxiliares Enfermeria & Total \\
\hline Profesión & 20 & 13 & 33 \\
\hline & & & \\
$\%$ resultados & $64,541,9 \%$ & $100 \%$ \\
\hline
\end{tabular}




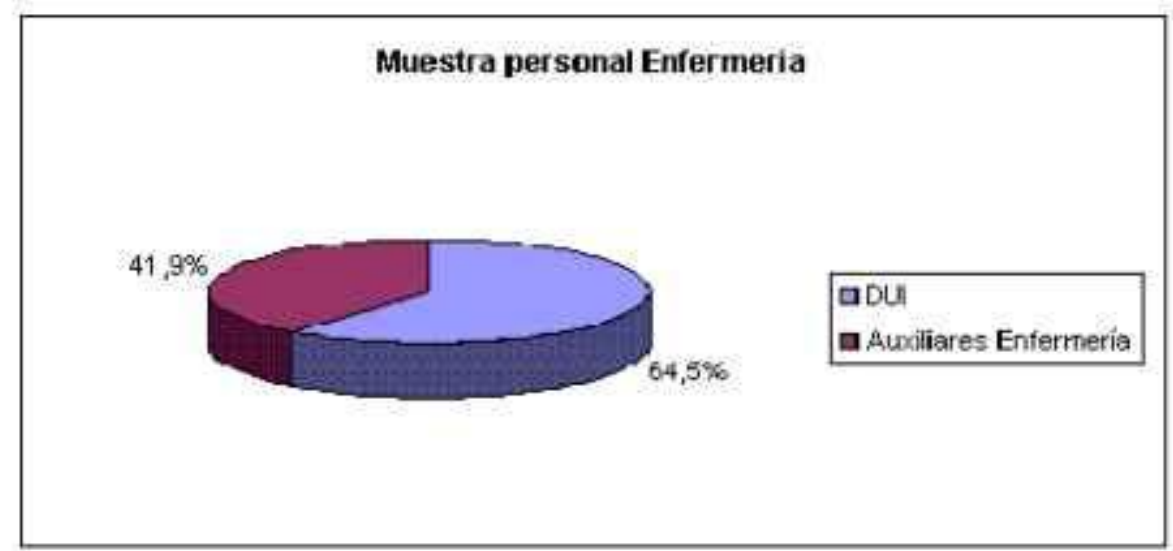

Gráfico 13

Tabla 14

\begin{tabular}{|l|l|l|l|l|}
\hline Edad & $20-30$ años & $30-40$ años & $40-50$ años & $50-60$ años \\
\hline Edad & 12 & 8 & 6 & 7 \\
\hline$\%$ resultados & $36 \%$ & $24 \%$ & $18 \%$ & $21 \%$ \\
\hline
\end{tabular}

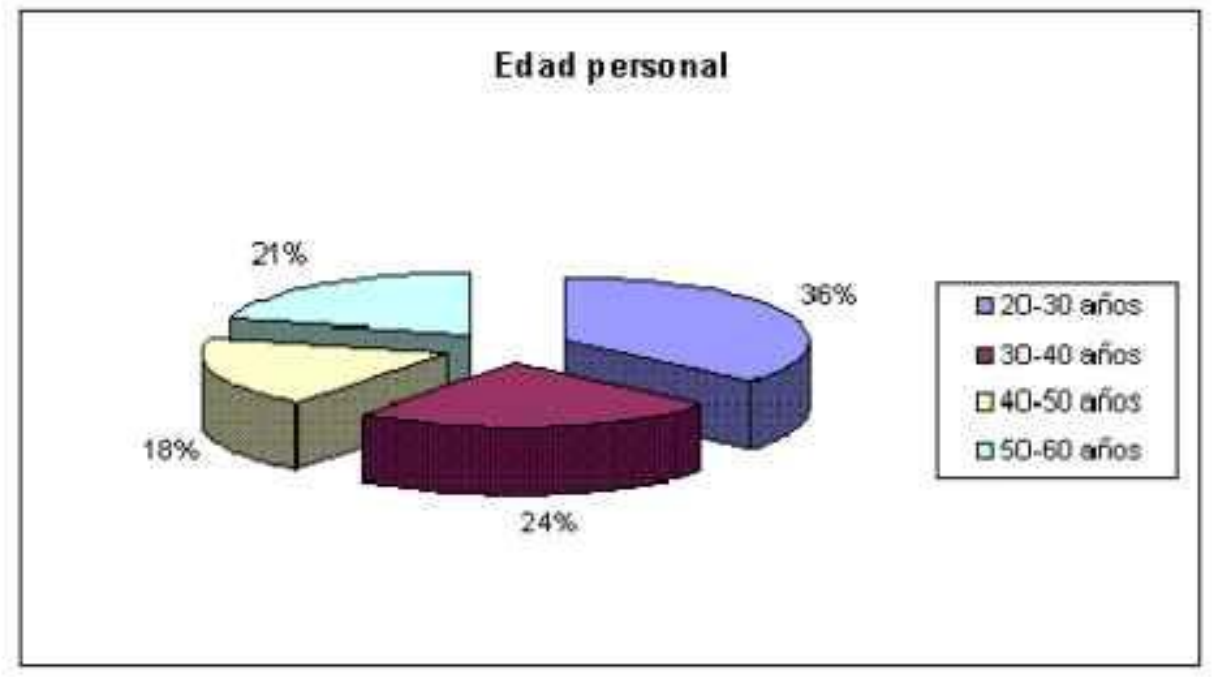

Gráfico 14 
En cuanto a la antigüedad de trabajo en la planta el $36 \%$ lleva de 6 meses a 2 años y de 5 a 10 años un $30 \%$.(tabla 15 y gráfico 15 ).

\section{Tabla 15}

\begin{tabular}{|l|l|l|l|l|}
\hline & 6meses-2 años & $2-5$ años & $5-10$ años & 10 años \\
\hline Antiguiedad & 12 & 6 & 10 & 5 \\
\hline$\%$ resultados & $36 \%$ & $18 \%$ & $30 \%$ & $15 \%$ \\
\hline
\end{tabular}

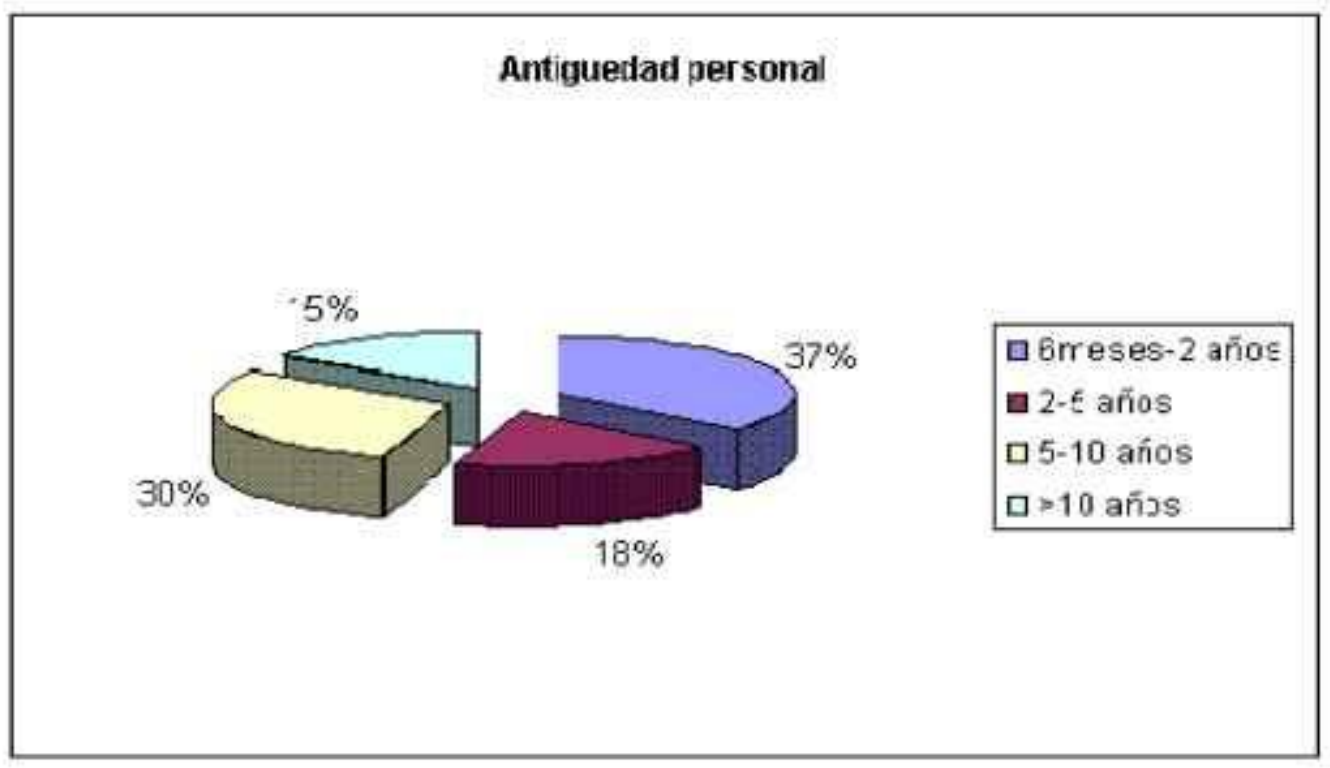

Gráfico 15

B.2.- DESCRIPCION DE LOS FACTORES QUE PERTURBAN EL SUEÑO SEGÚN EL PERSONAL DE ENFERMERIA

El $88 \%$ del personal de enfermería considera que los pacientes no descansan en el hospital. (tabla16 y gráfico 16).

\section{Factores de riesgo según enfermería}

Tabla 16

\begin{tabular}{|l|l|l|}
\hline & SI & NO \\
\hline Descansa & 4 & 29 \\
\hline$\%$ Descansa & $12 \%$ & $88 \%$ \\
\hline
\end{tabular}




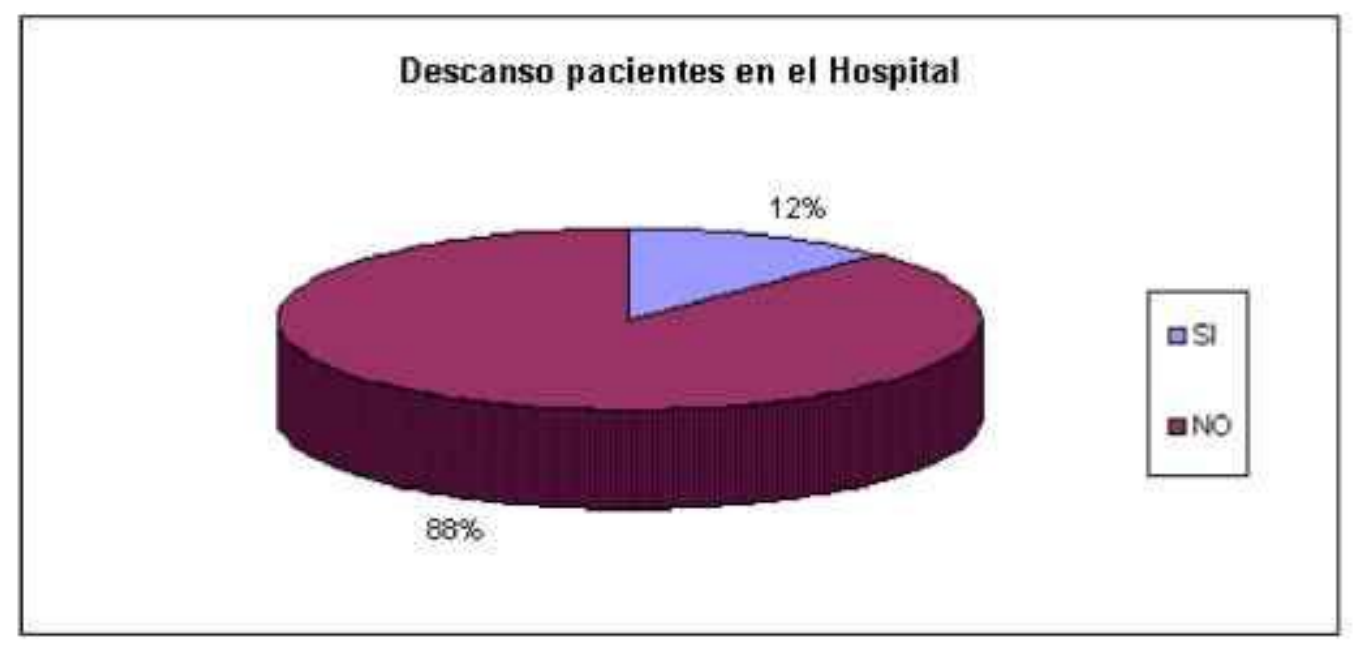

\section{Gráfico 16}

\section{FACTORES AMBIENTALES}

Analizando los distintos factores de riesgo encontramos que un $51,5 \%$ considera que los procedimientos de enfermería les afectan mucho y un $42 \%$ bastante. El ruido en la unidad un $61 \%$ consideran que les afecta bastante; la incomodidad de la cama un $48 \%$ bastante, un $27 \%$ mucho y en contrapartida un $21 \%$ poco.

Un 55\% del personal de enfermería considera que la iluminación les afecta bastante. El hecho de compartir habitación el $52 \%$ cree que les afecta bastante y un $36 \%$ mucho. (tabla y gráfico 17).

\section{Tabla17}

\begin{tabular}{|l|l|l|l|l|l|l|}
\hline Ambientales & Procedimientos & Ruido & Incomodidad & Iluminación & Compartir & Temperatura \\
\hline Nada & $0 \%$ & $0 \%$ & $3 \%$ & $15 \%$ & $0 \%$ & $0 \%$ \\
\hline Poco & $6 \%$ & $15 \%$ & $27 \%$ & $27 \%$ & $12 \%$ & $27 \%$ \\
\hline Bastante & $42 \%$ & $61 \%$ & $48 \%$ & $55 \%$ & $52 \%$ & $42 \%$ \\
\hline Mucho & $51,5 \%$ & $24 \%$ & $21 \%$ & $3 \%$ & $36 \%$ & $30 \%$ \\
\hline
\end{tabular}




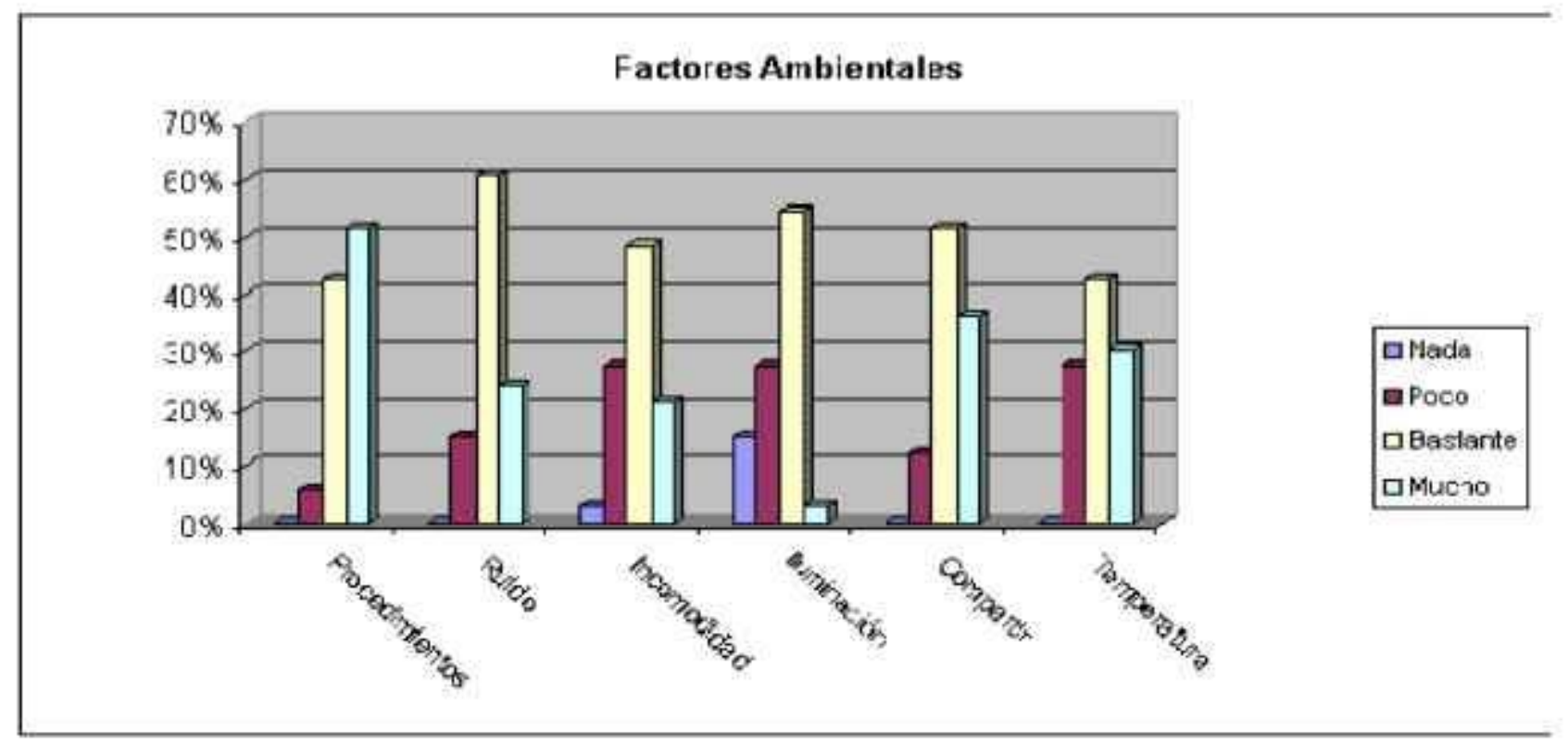

\section{Gráfico 17}

\section{FACTORES EXTERNOS}

Un $64 \%$ del personal de enfermería considera bastante influyente en el descanso el llevar sueros, así como el llevar sonda vesical (67\%), oxigenoterapia (64\%) y drenajes (58\%).El $42 \%$ del personal de enfermería cree bastante influyente en el sueño llevar SNG y un $42 \%$ lo considera muy influyente. (tabla 18 y gráfico 18 )

\section{Tabla 18}

\begin{tabular}{|l|l|l|l|l|l|}
\hline Factores Externos & Suero & SV & SNG & Oxigeno & Drenajes \\
\hline Nada & $0 \%$ & $3 \%$ & $3 \%$ & $0 \%$ & $3 \%$ \\
\hline Poco & $9 \%$ & $21 \%$ & $12 \%$ & $15 \%$ & $24 \%$ \\
\hline Bastante & $64 \%$ & $67 \%$ & $42 \%$ & $64 \%$ & $58 \%$ \\
\hline Mucho & $27 \%$ & $12 \%$ & $42 \%$ & $21 \%$ & $5 \%$ \\
\hline
\end{tabular}

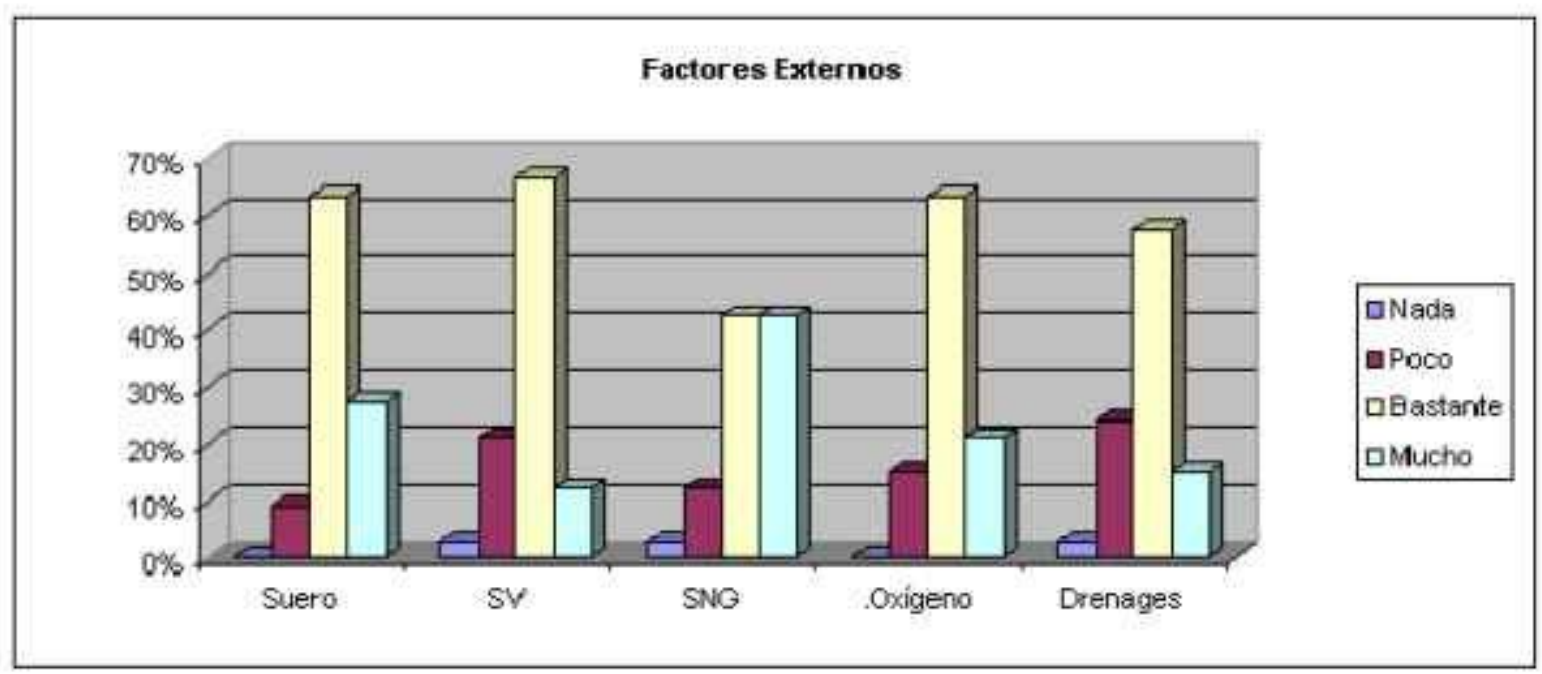




\section{FACTORES FISIOLÓGICOS}

Un $76 \%$ del personal de enfermería considera que el dolor perturba el sueño de los pacientes; el $48 \%$ la micción nocturna les afecta mucho; el $48 \%$ cree que les afecta mucho y el $39,4 \%$ bastante respecto a las nauseas y vómitos. Un $48,5 \%$ cree que la fiebre perturba bastante el descanso y un $42 \%$ mucho. La tos es un factor que el $67 \%$ de las encuestadas cree que puede afectar al sueño del paciente mucho.

Un $73 \%$ del personal de enfermería considera la disnea como un factor que afecta mucho a los pacientes. Las epigastralgias los consideran muy perturbador en mucho un $48 \%$; la movilidad en la cama lo considera un $51,5 \%$ en bastante y un $24 \%$ en mucho; el hambre/sed lo considera un $42 \%$ en poco y un $33,3 \%$ en bastante. (tabla y gráfico 19).

\section{Tabla 19}

\begin{tabular}{|l|l|l|l|l|}
\hline Factores fisiológicos & Nada & Poco & Bastante & Mucho \\
\hline Dolor & $0 \%$ & $3 \%$ & $33,3 \%$ & $76 \%$ \\
\hline Micción & $0 \%$ & $9 \%$ & $33,3 \%$ & $48 \%$ \\
\hline Vomitos & $0 \%$ & $12 \%$ & $39,4 \%$ & $48 \%$ \\
\hline Fiebre & $0 \%$ & $9 \%$ & $48,5 \%$ & $42 \%$ \\
\hline Tos & $0 \%$ & $6 \%$ & $27,3 \%$ & $67 \%$ \\
\hline Disnea & $0 \%$ & $6 \%$ & $21,2 \%$ & $73 \%$ \\
\hline Epigastrảlgias & $0 \%$ & $12 \%$ & $39,4 \%$ & $48 \%$ \\
\hline Mobilizazión & $0 \%$ & $24 \%$ & $51,5 \%$ & $24 \%$ \\
\hline Hambre/Sed & $15 \%$ & $42 \%$ & $33,3 \%$ & $9 \%$ \\
\hline
\end{tabular}

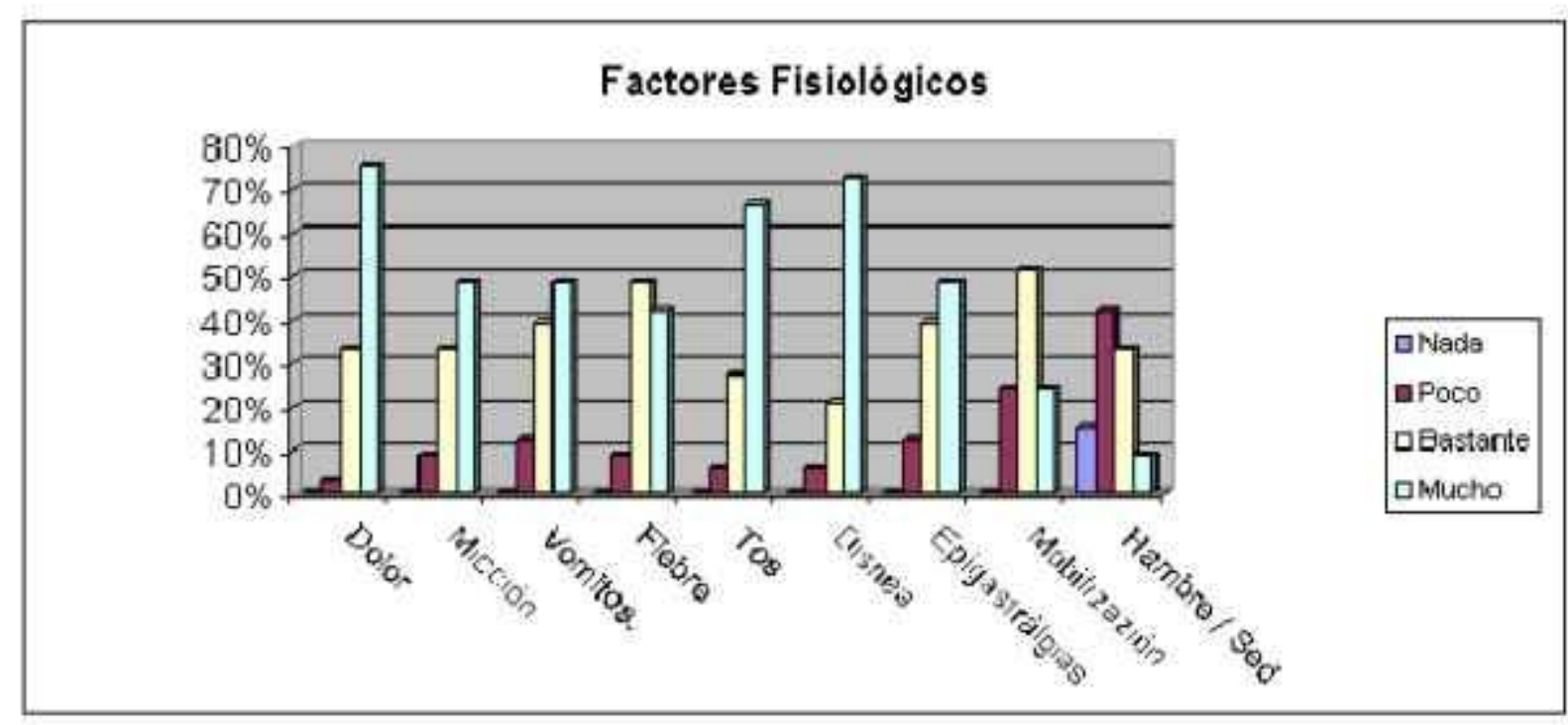

Gráfico 19 


\section{FACTORES PSICOLÓGICOS}

La preocupación por la enfermedad es considerada por el personal de enfermería muy influyente en un $63 \%$. Un $54 \%$ del personal considera que la preocupación por la familia les afecta en mucho; un $42 \%$ lo cree en bastante. En relación al trabajo, un $48,1 \%$ del las encuestados/as lo consideran un factor bastante influyente y un $42 \%$ lo califica de muy influyente. La ansiedad y depresión un $54 \%$ cree que mucho y un $45,2 \%$ cree que bastante. El aburrimiento el $27,1 \%$ del personal lo trata como un factor bastante influyente.

\section{4.- DISCUSIÓN}

Teniendo en cuenta que de los pacientes encuestados el $91 \%$ responde que se despierta durante la noche y de ellos el $26 \%$ lo hace de 2 a 3 veces y el $40 \%$ lo hace más de 3 veces, esto nos lleva a plantear si son más importantes las técnicas y el control nocturno a que sometemos a los pacientes o permitir su descanso, ya que la distribución del trabajo se hace en función del trabajo de enfermería y no en base al bienestar del paciente.

Da evidencia a estos resultados el hecho que un $53 \%$ respondió que dormía más de 7 horas en su casa. En el hospital solo el $28 \%$ descansa estas mismas horas.

Creemos que puede disminuir en el descanso nocturno el hecho que el $61 \%$ duerme también durante el día de 1 a 2 horas.

Destacamos que el $84 \%$ de los encuestados tienen diferentes hábitos y conductas para promover el buen dormir, donde destacan el ver la TV y la lectura.

Por otro lado, el $88 \%$ del personal de enfermería considera que el paciente no descansa durante su hospitalización.

Comparando los resultados de la muestra de enfermería y de los pacientes sobre los factores ambientales, en concreto sobre los procedimientos de enfermería, coinciden que les afecta bastante en el descanso. Aunque cabe destacar que un $31 \%$ de los pacientes contestó que no les afectaba nada. Esto podría ser debido a que el motivo del ingreso fuese por estudio, realización de pruebas diagnósticas. donde la atención de enfermería es menor que en un paciente agudo o crítico.

Coinciden también en el hecho de compartir habitación sea valorado como factor perturbador del sueño.

También queremos destacar que durante el tiempo de estudio hay una diferencia significativa entre lo que piensa el personal de enfermería y los pacientes en relación a los factores externos (llevar sueros, SV, SNG, drenajes), ya que enfermería considera que les afecta entre bastante y mucho y los pacientes lo califican como factor no perturbador del sueño. Quizás los resultados de la muestra de los pacientes son debidos a que muchos de ellos no han sido portadores de SV, sueros, SNG y por esta razón puntuaron como que no les afectaba en el sueño.

En lo referente a los factores fisiológicos (dolor, disnea, movilidad, nauseas-vómitos...), enfermería lo valora como algo bastante/ mucho perturbador del sueño y en cambio los pacientes lo valoraron en su mayoría como si no les afectara nada en su patrón de sueño/ descanso. Creemos que es debido a que por parte del equipo médico y enfermería hay una buena valoración y control de síntomas. 
Por último, los factores psicológicos son los más destacables e influyentes en el descanso; entre ellos la preocupación por la enfermedad y la familia y el aburrimiento durante la hospitalización. Coincidiendo mayoritariamente con los resultados del personal de enfermería.

\section{5.- CONCLUSIÓN}

Tanto enfermería como pacientes coinciden en un porcentaje elevado en que no se duerme en el hospital. Esto nos lleva a plantear la siguiente pregunta ¿Qué podemos hacer para que nuestros pacientes duerman mejor?

Teniendo en cuenta que se despiertan varias veces durante la noche deberíamos plantear:

- Reorganizar los cuidados para permitir el máximo sueño ininterrumpido posible

- Distinguir entre tareas fundamentales y no fundamentales

- Reducir al máximo el nivel de ruido en las unidades, ajustando el volumen de las alarmas de las bombas de perfusión y haciéndonos responsables de los efectos de nuestras conversaciones para el sueño de los pacientes.

Un aspecto a subrayar es que el personal de enfermería demostró tener una buena percepción de las causas principales de insomnio de los pacientes. Ambos han coincidido mucho en cómo los factores psicológicos influyen en el patrón de reposo/sueño.

Por ello sería importante que el personal de enfermería dedicara especial atención al manejo de la ansiedad, depresión y aburrimiento, enseñando ejercicios de relajación y creando talleres dirigidos a los pacientes (manualidades, música.) para favorecer contactos sociales.

En cuanto a la preocupación por su enfermedad, familia o trabajo se debería:

- Dialogar con el paciente para conocer y afrontar, en la medida de lo posible, sus preocupaciones y miedos.

- Enseñar técnicas de relajación

- Enseñar imaginaciones guiadas antes de ir a dormir, intentando que piense situaciones vividas que comporten un recuerdo agradable.

- Valoración psicológica y, si es preciso, tratamiento con terapia.

Con este estudio hemos pretendido demostrar que enfermería es un colectivo con identidad propia, capaz de realizar una importante labor dentro del equipo, encaminada sobretodo a conseguir una mejor calidad de vida de los pacientes.

\section{6. - BIBLIOGRAFIA}

1. Navarro JM, De Haro S, Orgiler P, Vela C: ¿Se respeta el sueño de los pacientes? Revista ROL Enf. (2001) 24:7-8

2. Novo Casal C, López Belda F, López Rodríguez MJ, Rombo Prieto JM, López Bellas $Z$, et al. Alteración del patrón reposo/sueño en ancianos hospitalizados. EC 1995;5(2): 49-53

3. Abad Massanet F, Arroyo López C. Insomnio en pacientes hospitalizados. Med Clin (Barc) 1992; 99:639 
4. Romero O, Sagales $\mathrm{T}$, Jurado MJ, Insomnio: Diagnostico, manejo y tratamiento. Rev. Med Univ Navarra / vol 49, $\mathrm{n}^{\circ} 1,2005 ; 25-30$

5. Cortes Angulo J, Travel García MC. El descanso de nuestros pacientes ¿se puede descansar en el hospital? Enfermería global n¹, nov 2002.

6. Ferrero Barrero M, García Díaz JC, Gómez García S, Rey San Millán MJ, Torres Sánchez V. Calidad del sueño del paciente oncológico. Comunicación oral presentada en XI Congreso nacional de la sociedad española de enfermería oncológica. Junio 2007.

7. Diagnósticos de enfermería: definiciones y clasificación 2005-2006. NANDA internacional. 


\section{ANEXO I}

\section{ENCUESTA PACIENTES}

Somos un grupo de enfermeras que queremos realizar un trabajo de investigación para conocer cómo es el sueño de los pacientes oncohematológicos durante la estancia en nuestro hospital; para ello necesitamos que rellene esta encuesta. Muchas gracias.

Edad Sexo: Hombre Mujer

Servicio donde está ingresado: Oncología

1. ¿Cuantas horas duerme en su casa? Hematología

2. ¿Y en el hospital? horas

3. ¿Toma algún medicamento para dormir? SI

4. ¿Duerme durante el día? SI NO i horas

5. ¿Se despierta durante la noche? SI uántas horas?

6. ¿Realiza alguna actividad antes de ir a dormir? SI__ NO Marque las actividades que usted realiza:

Escuchar la radio Mirar TV Leer Usar el ordenador Ninguna

7. Marque el número (grado) en que cree que le afectan cada una de las causas de la siguiente lista, donde el 0 significa que no le altera el sueño y el 10 altera totalmente el sueño.

Se corresponde 0-1: Nada; 2-4: Poco; 5-7: Bastante; 8-10: Mucho.

Factores ambientales:

- Interrupción por parte de enfermería para realizar procedimientos terapéuticos (toma de TA, temperatura, sueros.)

$\begin{array}{lllllllllll}0 & 1 & 2 & 3 & 4 & 5 & 6 & 7 & 8 & 9 & 10\end{array}$

- Ruido en la unidad (timbres, alarmas, carros.)

$\begin{array}{lllllllllll}0 & 1 & 2 & 3 & 4 & 5 & 6 & 7 & 8 & 9 & 10\end{array}$

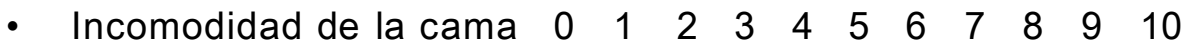

- Iluminación de la habitación $0 \begin{array}{lllllllllll}0 & 1 & 2 & 3 & 4 & 5 & 6 & 7 & 8 & 9 & 10\end{array}$

- Compartir la habitación $0 \begin{array}{lllllllllll}0 & 1 & 2 & 3 & 4 & 5 & 6 & 7 & 8 & 9 & 10\end{array}$

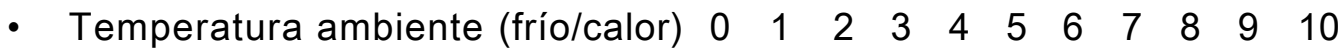

\section{Factores externos:}

- Sueroterapia $0 \begin{array}{lllllllllll}0 & 1 & 2 & 3 & 4 & 5 & 6 & 7 & 8 & 9 & 10\end{array}$

- Sonda vesical $0 \begin{array}{lllllllllll}0 & 1 & 2 & 3 & 4 & 5 & 6 & 7 & 8 & 9 & 10\end{array}$

- Sonda nasogástrica $0 \begin{array}{lllllllllll}0 & 1 & 2 & 3 & 4 & 5 & 6 & 7 & 8 & 9 & 10\end{array}$

- Máscara/gafas de oxígeno $0 \begin{array}{lllllllllll}0 & 1 & 2 & 3 & 4 & 5 & 6 & 7 & 8 & 9 & 10\end{array}$

- Drenajes $0 \begin{array}{lllllllllll}0 & 1 & 2 & 3 & 4 & 5 & 6 & 7 & 8 & 9 & 10\end{array}$

\section{Factores fisiológicos:}

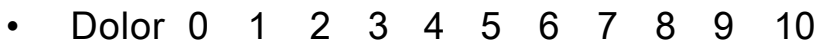

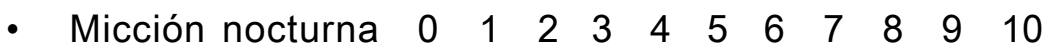

- Nauseas/ vómitos $0 \begin{array}{lllllllllll}0 & 1 & 2 & 3 & 4 & 5 & 6 & 7 & 8 & 9 & 10\end{array}$

- Fiebre $0 \begin{array}{lllllllllll}0 & 1 & 2 & 3 & 4 & 5 & 6 & 7 & 8 & 9 & 10\end{array}$

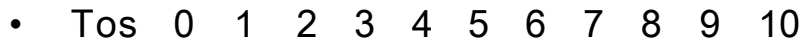

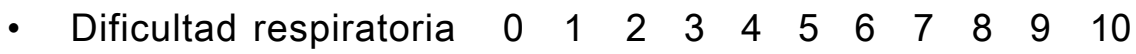

- Molestias gástricas $0 \begin{array}{lllllllllll}0 & 1 & 2 & 3 & 4 & 5 & 6 & 7 & 8 & 9 & 10\end{array}$

- Dificultad para movilizarse dentro de la cama $\begin{array}{lllllllllll}0 & 1 & 2 & 3 & 4 & 5 & 6 & 7 & 8 & 9 & 10\end{array}$

- Hambre y/o sed $0 \begin{array}{lllllllllll}0 & 1 & 2 & 3 & 4 & 5 & 6 & 7 & 8 & 9 & 10\end{array}$ 


\section{Factores psicológicos:}

- Preocupación por su enfermedad y/o hospitalización $\begin{array}{lllllllllll}0 & 1 & 2 & 3 & 4 & 5 & 6 & 7 & 8 & 9 & 10\end{array}$

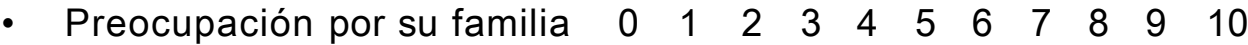

- Preocupación por su lugar de trabajo

$$
\begin{array}{lllllllllll}
0 & 1 & 2 & 3 & 4 & 5 & 6 & 7 & 8 & 9 & 10
\end{array}
$$

- Ansiedad/depresión $0 \begin{array}{lllllllllll}0 & 1 & 2 & 3 & 4 & 5 & 6 & 7 & 8 & 9 & 10\end{array}$

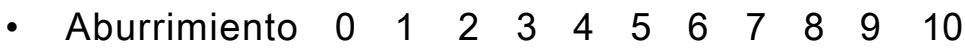




\section{ANEXO II}

Somos unas compañeras que trabajamos en el servicio de hematología y oncología que estamos realizando un estudio para conocer cómo duermen los pacientes oncohematológicos durante su estancia en nuestro hospital.

Agradecemos de antemano vuestra colaboración, rellenando la siguiente encuesta.

\section{ENCUESTA PERSONAL DE ENFERMERIA}

\section{Edad}

Categoría profesional: Auxiliar de enfermería

DUI

Antigüedad en el servicio: años

Turno: Mañana Tarde Noche

1) ¿Crees que el paciente descansa por la noche cuando está hospitalizado?

$\mathrm{SI}_{-} \mathrm{NO}_{-}$

2) ¿Cuales crees que son los factores que alteran el sueño de los pacientes?

Marque el número (grado) en que cree que le afectan cada una de las causas de la siguiente lista, donde el 0 significa que no le altera el sueño y el 10 significa que altera totalmente el sueño.

Se corresponde 0-1: Nada; 2-4: Poco; 5-7: Bastante; 8-10: Mucho.

\section{Factores ambientales:}

- Interrupción por parte de enfermería para realizar procedimientos terapéuticos (toma de TA, temperatura, sueros.)

$$
\begin{array}{llllllllllll}
0 & 1 & 2 & 3 & 4 & 5 & 6 & 7 & 8 & 9 & 10
\end{array}
$$

- Ruido en la unidad (timbres, alarmas, carros.)

$$
\begin{array}{lllllllllll}
0 & 1 & 2 & 3 & 4 & 5 & 6 & 7 & 8 & 9 & 10
\end{array}
$$

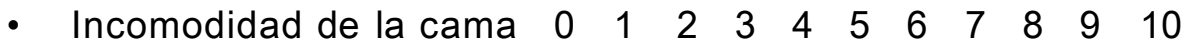

- Iluminación de la habitación $\begin{array}{llllllllllll}0 & 1 & 2 & 3 & 4 & 5 & 6 & 7 & 8 & 9 & 10\end{array}$

- Compartir la habitación $0 \begin{array}{lllllllllll}0 & 1 & 2 & 3 & 4 & 5 & 6 & 7 & 8 & 9 & 10\end{array}$

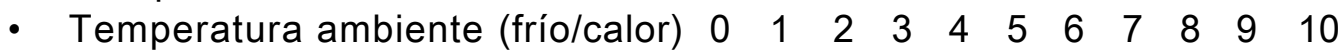

\section{Factores externos:}

- Sueroterapia $0 \begin{array}{lllllllllll}0 & 1 & 2 & 3 & 4 & 5 & 6 & 7 & 8 & 9 & 10\end{array}$

- Sonda vesical $0 \begin{array}{lllllllllll}0 & 1 & 2 & 3 & 4 & 5 & 6 & 7 & 8 & 9 & 10\end{array}$

- Sonda nasogástrica $0 \begin{array}{lllllllllll}0 & 1 & 2 & 3 & 4 & 5 & 6 & 7 & 8 & 9 & 10\end{array}$

- Máscara/ gafas de oxígeno $0 \begin{array}{lllllllllll}0 & 1 & 2 & 3 & 4 & 5 & 6 & 7 & 8 & 9 & 10\end{array}$

- Drenajes $0 \begin{array}{lllllllllll} & 1 & 2 & 3 & 4 & 5 & 6 & 7 & 8 & 9 & 10\end{array}$

\section{Factores fisiológicos:}

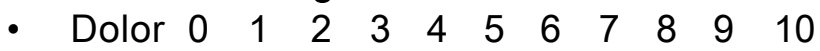

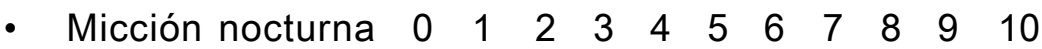

- Nauseas/ vómitos $0 \begin{array}{lllllllllll}0 & 1 & 2 & 3 & 4 & 5 & 6 & 7 & 8 & 9 & 10\end{array}$

- Fiebre $0 \begin{array}{lllllllllll}0 & 1 & 2 & 3 & 4 & 5 & 6 & 7 & 8 & 9 & 10\end{array}$

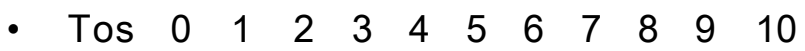

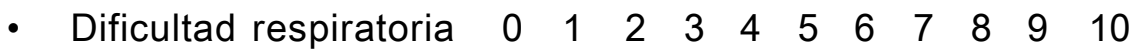

- Molestias gástricas $0 \begin{array}{lllllllllll}0 & 1 & 2 & 3 & 4 & 5 & 6 & 7 & 8 & 9 & 10\end{array}$

- Dificultad para movilizarse dentro de la cama $\begin{array}{lllllllllll}0 & 1 & 2 & 3 & 4 & 5 & 6 & 7 & 8 & 9 & 10\end{array}$

- Hambre y/o sed $0 \begin{array}{lllllllllll}0 & 1 & 2 & 3 & 4 & 5 & 6 & 7 & 8 & 9 & 10\end{array}$

\section{Factores psicológicos:}


- Preocupación por su enfermedad y/o hospitalización $\begin{array}{lllllllllll}0 & 1 & 2 & 3 & 4 & 5 & 6 & 7 & 8 & 9 & 10\end{array}$

- Preocupación por su familia $\begin{array}{llllllllllll}0 & 1 & 2 & 3 & 4 & 5 & 6 & 7 & 8 & 9 & 10\end{array}$

- Preocupación por su lugar de trabajo $\begin{array}{lllllllllll}0 & 1 & 2 & 3 & 4 & 5 & 6 & 7 & 8 & 9 & 10\end{array}$

- Ansiedad/depresión $\begin{array}{llllllllllll}0 & 1 & 2 & 3 & 4 & 5 & 6 & 7 & 8 & 9 & 10\end{array}$

- Aburrimiento $00 \begin{array}{llllllllll}0 & 2 & 3 & 4 & 5 & 6 & 7 & 8 & 9 & 10\end{array}$ 